\title{
Soap bubbles make serious physics
}

A new study of the evolution of rafts of soap bubbles confirms expectations and earlier measurements, but has the distinction of having been carried out with a photocopying machine.

Using; a Xerox or some other brand of photocopying machine, with virtually no extra equipment, to make interesting physical observations is an achievement in itself. But Joel Stavans from the University of Pittsburgh and James A. Glazier of the University of Chicago seem also to have won some interesting physics from their primitive equipment (Phys. Rev. Lett. 62, 1318; 1989). The issue is the evolution in time, by coalescence and other means, of a raft of soap bubbles which are small to begin with, but which get larger with the passage of time.

As with so many other topics, that of the evolution of a bubble raft goes back at least to J. von Neumann, among other things the author of a law describing the evolution of a single bubble embedded in a raft of them. It is important that, in cirumstances such as these, bubbles are not circular or spherical but, rather, sit like prisms on polygonal bases. The neat way of fixing ideas is to imagine the experiment which Stavans and Glazier describe: put a raft of coloured soap bubbles in a very shallow plastic tray and seal a lid on top in such a way that the soap films stretch between the flat and horizontal upper and lower surfaces, giving each bubble (to a first approximation) the same height.Liquid collects at the lower boundaries between contiguous bubbles, so that the evolution of the bubbles in a raft may be followed simply by pressing the button on the photocopier at convenient intervals.

A bubble will grow or shrink by the diffusion of supporting gas from all nearest neighbours, which leads directly to von Neumann's law of bubble-growth that the rate of change of the volume of a bubble (or of its area as seen in a photocopied image) depends only on the number of vertical faces it presents to its neighbours in the raft, or on the number of sides of the polygon which forms the base. The starting-point for the argument is that a strictly regular array of bubbles each of which is hexagonal should be indefinitely stable, which leads directly to the rule dal $\mathrm{d} t=\kappa(n-6)$, where $a$ is the projected area of the bubble, $n$ the number of sides and $\kappa$ a kind of diffusion constant.

The simplicity of this result is geometrical, deriving from the way in which both that rate of inward diffusion and the volume of the prism is determined by the total area of the vertical faces of the prismatic bubbles. A further assumption is that the internal angles of each polygonal bubble-base are exactly $120^{\circ}$, which seems reasonable enough, given that each vertex of the polygon is a point at which three prismatic bubbles join; to a first approximation, they may be assumed to join symmetrically. But naturally this can be exactly true only when all bubble bases are regular hexagons. Otherwise, the sides of the polygonal bases will not be straight lines, but curves.

Stavans and Glazier are not primarily concerned with single bubbles, but with rafts of them, for which purpose they work with a distribution function called $\varrho(n)$ which gives the frequency in any sample of the evolving bubble raft of bubbles with $n$ sides, where $n$ is necessarily an integer running from 3 to infinity.

Simple observation is said to show that the first stages of the evolution of the raft are rapid, with larger bubbles growing at the expense of smaller neighbours and the sidedness of particular bubbles changing upwards and downwards both by the disappearence of smaller neighbours and by a process in which vertical membranes shift so as to trade sides between one bubble and a neighbour. (Those planning to use their office photocopier for further investigations along these lines should beware of the edge effects, which complicate both the statistics and the underlying phenomenon.)

The authors explain how, when they made soap bubbles with helium gas, the general appearence of the raft thus formed was that of several patches of regular hexagonal bubbles interspersed with regions in which five and seven-sided polygons were mixed together. But the striking result, confirming earlier conjectures, is that there comes a point in the evolution of a bubble-raft in which the distribution function $\varrho(n)$ becomes essentially invariant in the course of time. Moreover, the mean of the distribution (that is the average number of sides per bubble) is almost but not quite six, whatever the length of time for which the raft has been evolving (in one run, indeed, measurements extended over a day and a half). Only when the total number of bubbles becomes very small does the distribution go awry.

The same point emerges from measurements of the second moment of the distribution (essentially the weighted average of the squared deviation of the number of sides per cell from the average value). Again, in at least two different systems, the value of the second moment fluctuated widely to begin with, but then quickly settled down to what seems to have been a constant value (estimated at $1.4 \pm 0.4)$. The interest seems to be that. as in fractal and chaotic systems, there seems to be an underlying scaling law which describes how, in an ageing raft of bubbles, the bubbles all get bigger, but the statistical properties of the quantity appropriately called sidedness remain essentially constant.

Departures from the rule that the internal angles of the basal polygons of each bubble should be $120^{\circ}$ excite particular interest. What the measurements show is that bubbles with fewer than six sides have internal angles smaller or equal to $120^{\circ}$, but that bubbles with more sides have larger angles (although $130^{\circ}$ seems to be the limit). Stavans and Glazier argue that the presence of angles differing from the expected value implies the accumulation of strain energy in the system, which allows them to generalize von Neumann's law (but only for those systems in which all the bubbles have the same number of sides) to take account of these departures from the expected. One practical conclusion seems to be that it is possible to understand why one bubble may grow at the expense of a neighbour with the same number of sides.

There remains one puzzle. Perhaps the simplest measure of the state of an ageing bubble raft is the average bubble area, which should, by simple integration of von Neumann's rule, be proportional to the time elapsed. But neither Stavans and Glazier nor their predecessors in the field have been able to measure anything like that - they quote a figure of $0.59 \pm 0.11$. as the exponent in a simple power-law dependence on the time.

Why should such a simple prediction apparently be so wide of the mark? It is natural that people should have gone haring after fanciful explanations to do with the scaling laws as bubble-rafts age. Prosaically, the authors suggest a more mundane explanation - that as a raft ages, more soap solution accumulates beneath the raft while there are thicker layers of it between membranes, the effect of which is to reduce the diffusion constant between neighbouring bubbles. It all goes to show that there are merits in even the least reliable photocopiers

John Maddox 